\title{
Design Issues of Spectrum Sensing in Cognitive Radio Networks
}

\author{
Bub-Joo Kang, Member, KIMICS
}

\begin{abstract}
This paper investigates the design issues of spectrum sensing in the cognitive radio (CR) networks of opportunistic unlicensed spectrum access. The cognitive radios can perform a communication using the incumbent user spectrum band without the interference caused by the cognitive radio users. In this case, the cognitive radios must know the real-time radio environments of the incumbent user spectrum band using the spectrum sensing, beacon signal, and geo-location database access. Then in this paper, we are going to provide spectrum sensing issues which include the sensing techniques, the regulatory requirements, the analysis of DTV detection threshold, and main considerations associated with the spectrum sensing design in cognitive radio systems. Also, this paper introduces design trade-offs in order to optimize the sensing parameters such as sensing time and sensing complexity.
\end{abstract}

Index Terms - Spectrum sensing, detection method, sensing requirements, cognitive radio $(C R)$.

\section{INTRODUCTION}

THE licensed spectrum allocation method appears to have low spectrum utilization in many parts of the frequency band. In the Federal Communications Commission (FCC) definition, a cognitive radio (CR) system is a spectrum utilization that allows CR users to use spectrum licensed to incumbent users when they are inactive [1],[2]. In order to utilize the inactive frequency bands, CR users must perform the spectrum sensing for the CR-used frequency bands frequently or periodically. The main reasons for spectrum sensing in CR users can be classified into two types. The first is to gather vacant channels for CR users, and the second is to check the appearance of incumbent users for the corresponding channel that a CR user is utilizing.

The methods knowing the current spectrum usage are three kinds of spectrum sensing, geo-location database access, and beacon signal like a cognitive pilot channel [3]. The geo-location database access needs the

Manuscript received March 29, 2011; revised April 4, 2011; accepted April 15, 2011.

Bub-Joo Kang is with the Department of Information and Communication Engineering, Dongguk University, Gyeongju, 780-714, Korea (Email: bjkang@dongguk.ac.kr) infrastructure and internet connection to access the spectrum database. The beacon signal needs the standardization control channel to broadcast the beacons and channel availability states in cell coverage. Also their implementation requires positioning function to the $\mathrm{CR}$ users. On the other hand, spectrum sensing depends on the CR system to identify the vacant channels through spectrum sensing of the incumbent bands. In this case the transceiver hardware of the CR system becomes more complex than the previous two methods because of the complexity of spectrum sensing.

In this paper, we suggest an overview of the sensing techniques, cooperative spectrum sensing, sensing requirements of IEEE 802.22, comparison between geolocation and spectrum sensing requirements related to the regulatory policy of both FCC and wireless regional area network (WRAN), and sensing optimization.

This paper is organized as follows. In section II, we survey design issues of the direct spectrum sensing and finally conclusions are offered in section III.

\section{DESIGN ISSUES}

In this section, this paper handles the design issues of the direct spectrum sensing to obtain the observation results such as the identification of white spaces and appearance of incumbent users in an opportunistic spectrum access system. The main items surveyed in this paper are the spectrum sensing techniques, cooperative spectrum sensing, sensing requirements of IEEE 802.22, comparison between geo-location and spectrum sensing requirements, and sensing optimization.

\section{A. Spectrum Sensing Techniques}

The main sensing methods are known as the energy detection (ED), maximum eigenvalue (ME)-based detection, matched filtering (MF)-based detection, and cyclostationary feature detection (CFD), which have different requirements, advantages, and disadvantages. The energy detection and maximum eigenvalue detection are the blind detection methods that do not require any information of the incumbent signal while the matched filtering and cyclostationary feature detection require the perfect information of the incumbent signal.

At first, the energy detection is the most general 
method due to its low computational and implementation complexities. The incumbent signal is detected by the output of energy detector with a threshold which depends on the noise variance. So, the problems of the energy detection are mentioned as the optimal threshold selection, inability to differentiate the received signal from incumbent users and interference noise [4].

The maximum eigenvalue detection is known as a method to overcome the drawback of the energy detection using autocorrelations of the received signal [5]. Actually the interference noise level changes with time, which yields noise uncertainty. There are two types of noise uncertainty generated by receiver device noise and channel environment noise. The noise uncertainty caused by a receiver device comes from the nonlinearity and the thermal noise of device components. The channel environment noise uncertainty is caused by the other user interferences. In this case, it is difficult to estimate the accurate noise variance. Thus, the energy detection method has the disadvantages in terms of the optimal threshold selection due to noise uncertainty. However, in maximum eigenvalue detection method, the threshold related to false alarm probability can be selected by either theoretical derivation or computer simulation regardless of any information of channel noise power. The autocorrelations of the received signal and noise are different. Thus this difference is used in the method to differentiate the incumbent signal from the background noise. The steps of the maximum eigenvalue detection method are as follows: First, the sample covariance matrix of the received signal is calculated using the received signal samples. Then, two test statistics are extracted from the sample covariance matrix. Finally, the decision for the presence of the incumbent signal is performed by comparing the ratio of two test statistics with the threshold.

The matched filtering is known as the optimum detection method for the incumbent signal when the incumbent signal is known. So, the sensing performance of the matched filtering detection is superior to other detection methods. However, the matched filtering detection requires the perfect information of the incumbent signal such as bandwidth, carrier frequency, modulation, pulse shaping, and frame format to demodulate the received signal. Also, because the spectrum sensor has receivers for all signal types, the implementation complexity is very large.

The cyclostationary feature detection is a method for detecting the incumbent signal by utilizing the cyclostationary features of the received signal. The cyclostationary features are given by the periodicity in the signal. So, the cyclostationary feature detection requires the perfect information for the cycle frequencies of the incumbent signals.

Table I shows comparisons of detection methods in terms of complexity, noise uncertainty, and blind detection. Then the optimum detection method according to characteristics of a CR system is selected by considering design trade-offs such as the detector complexity, information of an incumbent signal, required detection threshold, radio channel environments, and required channel detection time.

TABLE I

COMPARISONS OF DETECTION METHODS

\begin{tabular}{|c|c|c|c|}
\hline Detection Method & Complexity & $\begin{array}{c}\text { Noise } \\
\text { Uncertainty }\end{array}$ & $\begin{array}{c}\text { Blind/ } \\
\text { Specific }\end{array}$ \\
\hline Energy Detection & Low & High & Blind \\
\hline $\begin{array}{c}\text { Maximum } \\
\text { Eigenvalue }\end{array}$ & High & Low & Blind \\
\hline $\begin{array}{c}\text { Cyclostationary } \\
\text { Feature }\end{array}$ & High & Low & Specific \\
\hline Matched Filtering & High & Medium & Specific \\
\hline
\end{tabular}

\section{B. Cooperative Spectrum Sensing}

In general the cooperative spectrum sensing is known as a solution to problems that occur in spectrum sensing due to fading, shadowing, and hidden node. Thus the probability of miss detection and the probability of false alarm are decreased by the cooperative spectrum sensing.

The cooperative spectrum sensing can be comprised of two schemes in terms of the collection method of sensing information. One is the centralized spectrum sensing, the other is the distributed spectrum sensing. In the centralized spectrum sensing, a central node such as a base station (BS) or an access point (AP) collects the sensing information for the sensors and determines the vacant channels, and then broadcasts this information to other cognitive radios. Thus the central node determines all the functions such as channel search, channel move, and identification of an incumbent user using the sensing information collected by the spectrum sensors. In the case of the distributed spectrum sensing, the spectrum sensors share the sensing information among each other but the final decisions for channel move and channel assignment are performed by themselves without any control of a central node. So the distributed spectrum sensing is more advantageous in the view that does not require a backbone infrastructure [4].

Also the cooperative spectrum sensing can be classified by the combining methods of the measurements of the sensing nodes, which are known as hard decision combining and soft decision combining [6],[7]. A hard decision combining means that each sensor measures the signal energy in 
spectrum band, and then compares it to a detection threshold and makes a decision on the presence of an incumbent user. In the distributed spectrum sensing, each sensor node shares its decision with other sensor nodes using zero or one to inform whether they observe a vacant channel or an occupied channel, respectively. The decisions collected from cooperating sensor nodes can be combined in two ways, which are an OR rule and an AND rule. The decision of an OR rule is that an incumbent user is present if one of the sensing nodes detects an incumbent user. On the contrary, that of an AND rule is that an incumbent user is present if all the sensing nodes detect an incumbent user. And the soft decision combing means that information on the reliability of the measurement is included by sharing more information on the measured signal energy between the sensing nodes than a immediate decision whether the channel is vacant or occupied. If no quantization is used, the sensing nodes send their full observations of the signal energy to the cognitive radio requesting sensing information. In the case of the centralized spectrum sensing, the central node sums up the measurements from the sensing nodes and determines whether they observe a vacant channel or an occupied channel by comparing the sum to a detection threshold.

\section{Sensing Requirements}

The opportunistic spectrum access of the CR users is made by guaranteeing the protection of an incumbent user from harmful interference. Thus, in order to protect an incumbent user from harmful interference, the incumbent users require the spectrum sensor to satisfy the following sensing requirements. In this paper, the sensing requirements are written by referring to IEEE 802.22 functional requirement document [1].

The sensing requirements for the WRAN users are mainly comprised of the dynamic frequency selection (DFS) time parameters and incumbent detection thresholds to satisfy the required miss detection probability and false alarm probability.

First of all, the incumbent detection thresholds required in WRAN are described below. Currently the incumbent systems within TV band are analog TV, advanced television system committee digital TV (ATSC DTV), and wireless microphone. DTV threshold is $-116 \mathrm{dBm}$ as a total ATSC DTV power in the $6 \mathrm{MHz}$ channel. Analog TV threshold is $-94 \mathrm{dBm}$ as a power measured at a synchronized peak of the national television system committee (NTSC) picture carrier. And, wireless microphone threshold is $-107 \mathrm{dBm}$ as a power measured in $200 \mathrm{kHz}$ bandwidth.

Secondly, the dynamic frequency selection (DFS) timing requirements to avoid interference to incumbent users from WRAN system operation are as follows [1]. The channel availability check time is the time during which a TV channel shall be checked for the presence of incumbent users prior to the beginning of WRAN operation in that channel. The non-occupancy period is the required time during which WRAN device transmissions shall not be allowed in a given TV channel due to the detected presence of an incumbent signal in that channel. The channel detection time is the maximum time for a WRAN device to detect a licensed incumbent signal, when the incumbent signal appears in a given TV channel during normal WRAN operation. The channel setup time is the channel connection time that may be taken by a WRAN device to transmit control information to a WRAN base station in order to establish operation with that base station at the prescribed power, in the case of TV, below the allowable effective isotropic radiated power (EIRP) within a given TV channel. The channel opening transmission time is the aggregate duration of control transmissions by WRAN devices during the channel setup time. The channel move time is the time taken by a WRAN system to stop all interfering transmissions on the given TV channel due to detection of an incumbent signal. The channel closing transmission time is the aggregate duration of control transmissions by the WRAN devices during the channel move time in order to vacate the given TV channel due to detection of an incumbent signal. Finally, in-service monitoring is a mechanism to monitor an operating TV channel in use by WRAN devices for the appearance or presence of an incumbent signal above the relevant incumbent detection threshold during normal WRAN operation.

The important DFS timing parameters are channel detection time and channel move time. The channel detection time is the timing parameter related to design the sensing period and sensing time. Here the sensing period is in inverse proportion to the sensing repetition rate in service monitoring and the sensing time is determined by the signal detection time of the spectrum sensor. Thus the sensing period and sensing time have an effect on data throughput of a CR user. And the shortening of the channel move time has an influence on the reduction of the CR user interference as well as the increase of the $\mathrm{CR}$ data throughput.

TABLE II

WRAN DFS TIMING PARAMETERS [1]

\begin{tabular}{|l|l|l|}
\hline DFS Parameter & $\begin{array}{l}\text { Value for Part 74 } \\
\text { Devices }\end{array}$ & $\begin{array}{l}\text { Value for TV } \\
\text { Broadcasting }\end{array}$ \\
\hline $\begin{array}{l}\text { Channel Detection } \\
\text { Time }\end{array}$ & $\begin{array}{l}<=2 \mathrm{sec} \text { to }>=90 \% \\
\text { Probability of } \\
\text { Detection with a } \\
\text { False Alarm rate of } \\
<=10 \%\end{array}$ & $\begin{array}{l}<=2 \mathrm{sec} \text { to }>=90 \% \\
\text { Probability of } \\
\text { Detection with a } \\
\text { False Alarm rate } \\
\text { of }<=10 \%\end{array}$ \\
\hline Channel Move Time & $2 \mathrm{sec}$ & $2 \mathrm{sec}$ \\
\hline $\begin{array}{l}\text { Incumbent } \\
\text { Detection Threshold }\end{array}$ & $\begin{array}{l}-107 \mathrm{dBm}(200 \mathrm{kHz} \\
\mathrm{BW})\end{array}$ & $\begin{array}{l}-116 \mathrm{dBm}(6 \mathrm{MHz} \\
\text { BW }) \text { for DTV }\end{array}$ \\
\hline
\end{tabular}


D. Comparison between Geo-location and Spectrum Sensing Requirements

In WRAN system, the sensing threshold for the digital TV is $-116 \mathrm{dBm}$. Then the thermal noise in $6 \mathrm{MHz}$ is approximately $-106.2 \mathrm{dBm}$. If the receiver noise figure assumes $10 \mathrm{~dB}$, the noise power becomes $-96.2 \mathrm{dBm}$. So, the receive power of $-116 \mathrm{dBm}$ is lower $19.8 \mathrm{~dB}$ than the thermal noise power. In this case, there are no spectrum sensors that detect an incumbent signal perfectly at the receive power level of $-116 \mathrm{dBm}$. Thus we think that the sensing thresholds for incumbent systems should be amended in future.

The FCC defines the protection contour for DTV around a TV tower as a contour of locations which the propagation model predicts that the $\mathrm{F}(50,90)$ field strength is $41 \mathrm{dBu}[8]$. The $\mathrm{F}(50,90)$ is the value that the field strength in $50 \%$ of locations and $90 \%$ of time is higher than that value $(41 \mathrm{dBu})$. This contour is a circle with a given radius centered at the TV tower. In order to know a radius of the TV protection contour, Fig. 1 can be utilized. Actually, the transmit power of DTV station is 1 MW (90 dBm). When the DTV antenna height is $500 \mathrm{~m}$ and the DTV operates at $615 \mathrm{MHz}$ in the UHF band, Fig. 1 shows the field strength versus distance for the $\mathrm{F}(50,90)$ curve in ITU channel model.

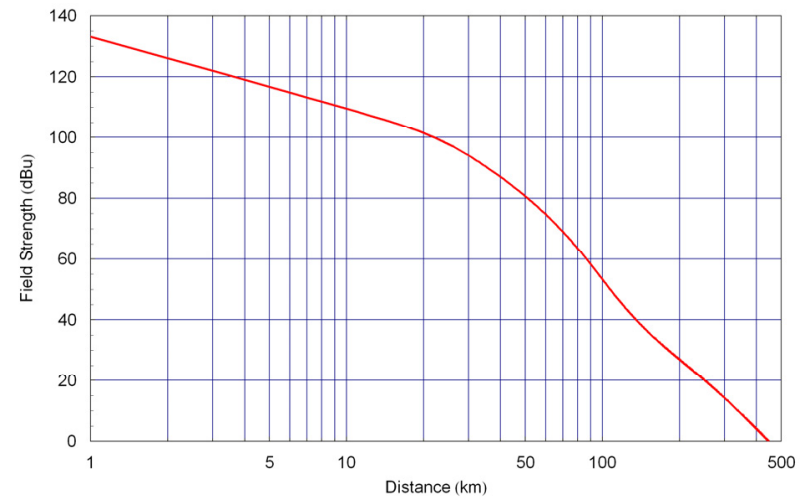

Fig.1. DTV field strength versus distance [8].

From Fig. 1, the radius of the protection contour is approximately $132 \mathrm{~km}$. In order to analyze the relation between geo-location and sensing requirements, this paper converts the propagation model from the field strength to the receive power. The equation for the conversion from the field strength to the receive power is represented by [9]

$$
\begin{aligned}
P_{d B m} & =20 \log (E)+10 \log (G)+20 \log (\lambda) \\
& -10 \log (120 \pi)-10 \log (4 \pi) \\
& =F_{d B \mu}+G_{d B}+20 \log (\lambda)-126.76
\end{aligned}
$$

where $P_{d B m}$ is the receive power in $\mathrm{dBm}, F_{d B \mu}$ is the field strength in $\mathrm{dBu}, G_{d B}$ is the receive antenna gain in
$\mathrm{dB}$, and $\lambda$ is the wavelength in meters. If the receive antenna gain and the operating frequency are $0 \mathrm{~dB}$ and $615 \mathrm{MHz}$ respectively, the conversion from the field strength to the receive power is approximately $-133 \mathrm{~dB}$ from the calculation of (1). So, the conversion equation from the field strength to the receive power is simplified by

$$
P_{d B m}=F_{d B \mu}-133
$$

Using (2), $F(50,90)$ curve is represented by the graph of DTV receive power versus distance as shown in Fig. 2.

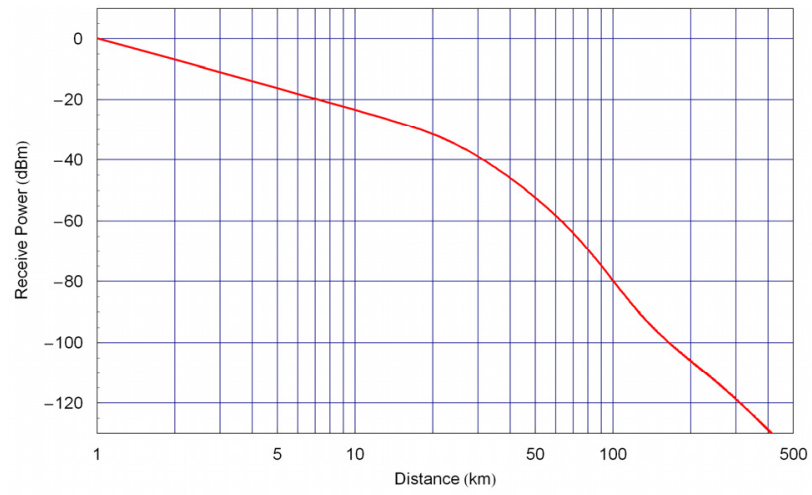

Fig. 2. DTV receive power versus distance [8].

From Fig. 1, we know that the radius of the protection contour is approximately $132 \mathrm{~km}$. In the case of Fig. 3, we intend to measure the radius of the keep-out region.

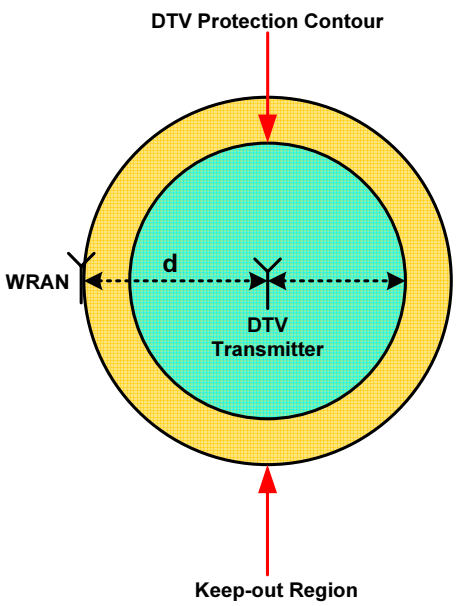

Fig. 3. WRAN base station at the edge of the keep-out region [8].

According to the FCC notice of proposed rulemaking (NPRM), the desired/undesired (D/U) ratio is $23 \mathrm{~dB}$. Here the desired signal is DTV signal and the undesired signal is WRAN signal. If the $F(50,90)$ field strength at DTV protection contour is $41 \mathrm{dBu}$, the undesired signal level needs to be $18 \mathrm{dBu}(41 \mathrm{dBu}-23 \mathrm{~dB})$ or less. From Fig.4, 
the distance which the field strength of the undesired signal reaches $18 \mathrm{dBu}$ is approximately $23 \mathrm{~km}$. Adding 23 $\mathrm{km}$ to the DTV protection contour of $132 \mathrm{~km}$ we obtain a keep-out region of $155 \mathrm{~km}$ around the DTV transmitter. At the edge of the keep-out region of $155 \mathrm{~km}$ from the DTV transmitter, the DTV field strength using the $\mathrm{F}(50,90)$ curve is $35.1 \mathrm{dBu}$ [8]. And the receive power assuming isotropic sensing antenna is $-97.9 \mathrm{dBm}$.

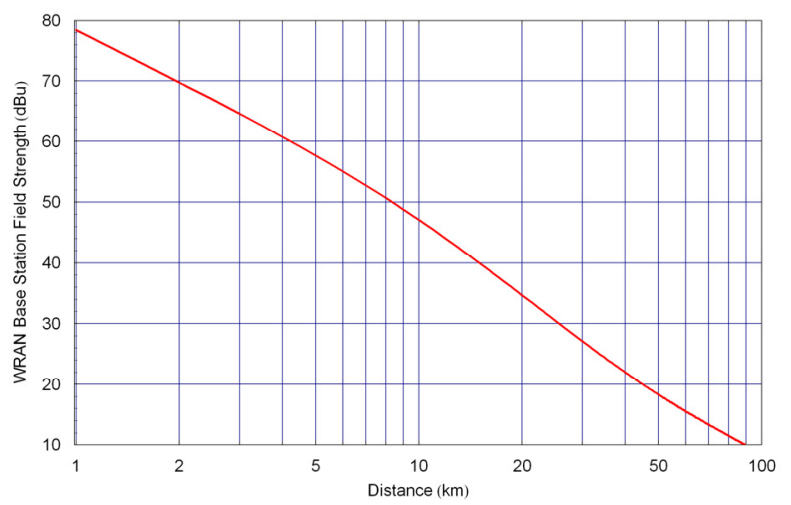

Fig. 4. WRAN base station field strength [8].

In table II, the incumbent detection threshold of DTV is $-116 \mathrm{dBm}$. If the receive power of DTV is $-116 \mathrm{dBm}$, the distance from the TV transmitter is approximately $280 \mathrm{~km}$ as shown in Fig. 2. The distance of $280 \mathrm{~km}$ corresponding to the receive power of $-116 \mathrm{dBm}$ is longer than that of the BS keep-out region of $155 \mathrm{~km}$. As a result, there are the large difference between BS keep-out region (geolocation requirement of FCC) and DTV spectrum sensing region (sensing requirement of WRAN). So, based on the BS keep-out region and DTV spectrum sensing region of table III, we think that the incumbent detection threshold of DTV will be modified as a value of $-98 \mathrm{dBm}$ or less in future.

TABLE III

DISTANCES FROM TV TRANSMITTER

\begin{tabular}{|l|l|}
\hline Protection Regions & Distance \\
\hline DTV Protection Contour & $132 \mathrm{~km}$ \\
\hline Base Station Keep-out Region & $155 \mathrm{~km}$ \\
\hline DTV Spectrum Sensing Region & $280 \mathrm{~km}$ \\
\hline
\end{tabular}

\section{E. Sensing Optimization}

In this part, this paper mentions the major trade-offs involved in the design of spectrum sensing functionality in the cognitive radio networks. The sensing designer should consider design trade-offs and find the optimal parameters according to the cooperation-detector complexity, sensing RF structure, and data throughput.

In cooperation-processing trade-off [6], the cooperation overhead generally increases with the number of cooperating CR users due to the increased volume of data that is reported to the spectrum manager and processed by it. Therefore, there exists a trade-off between the local detector processing overhead and the cooperation overhead as they add to the total sensing time. This tradeoff may be balanced by finding the optimum levels of detector-processing and cooperation, minimizing the total sensing overhead.

The trade-off between sensing time and data throughput in a CR system is an important design issue for sensing optimization. Because there is a trade-off between channel monitoring time and data transmission time, the advanced researches on the optimal sensing time have been performed in terms of throughput or throughput efficiency [10],[11]. One of two referred papers handles the problem of designing the sensing slot duration to maximize the achievable throughput for the $\mathrm{CR}$ users under the constraint that the incumbent users are sufficiently protected [10]. Using energy detection scheme, it shows that there is an optimal sensing time in terms of throughput. Cooperative sensing using multiple secondary users and diversity reception using multiple mini-slots are also studied based on the proposed tradeoff methodology. Also, other paper handles that the optimum sensing times for channel search and channel monitoring were obtained in order to maximize the average throughput of the CR user while protecting the incumbent user from harmful interference [11].

Another paper for sensing optimization evaluates the throughput of a CR user by considering such situations as the penalty time in the channel search process and an incumbent user (IU) detection delay in the channel monitoring process [12]. Also it derives the mean channel search time in consideration of the penalty time, which is caused by the channel search error created in the search process of a vacant channel. Also, if the CR system does not detect incumbent users during $\mathrm{CR}$ operation during the existence of an incumbent signal, it introduces the IU detection delay as the delay time caused by the continuous channel monitoring due to a misdetection. When the CR system continues to perform a CR operation during the existence of incumbent users, both the $\mathrm{CR}$ and incumbent users are affected by interference from each other. In such an event, it assumes that transmission errors in CR systems can occur without a successful data reception. Thus the throughput of the CR system in this case is almost zero. It also considers a sensing structure with two separate RF paths for the data transceiver and sensing receiver, and compares its sensing performance with that of a single RF path in terms of CR system throughput.

\section{CONCLUSIONS}

After finishing the second test of white space device (WSD), the FCC allowed personal/portable device operation with interference avoidance capability on TV white spaces using spectrum sensing function [13]. The 
decisions related to spectrum sensing are as follows. The fixed devices must include geo-location capability to inquire and download a coverage map database to determine available channels for $\mathrm{CR}$ operation in that location. Database service will be offered by a third party or parties. All CR devices must include spectrum sensing capability to identify the DTV, WM and other TV broadcast (TVBD) signals. There are two types of personal or portable devices. The mode I device is under control of a device that employs geo-location database access. Then the mode II device employs its own geolocation database access. Though sensing only personal/ portable devices will be allowed, those will be type approved, initially certified by the FCC regulation. Thus the CR device using the white space must have the interference avoidance capability such as the spectrum sensing and geo-location database access.

Based on the WRAN standardization and FCC regulation, this paper provides an overview of the design issues such as the spectrum sensing techniques, cooperative spectrum sensing, regulatory requirements, incumbent detection thresholds, sensing optimization, and other things related to the direct spectrum sensing.

\section{ACKNOWLEDGMENT}

This research was financially supported by the Ministry of Education, Science Technology (MEST) and Korea Institute for Advancement of Technology (KIAT) through the Human Resource Training Project for Regional Innovation.

\section{REFERENCES}

[1] IEEE 802.22, Functional Requirements for the 802.22 WRAN Standard, IEEE 802.22-05/007r46, September 2005.

[2] IEEE 802.22 WG, Draft standard for wireless regional area networks part 22, IEEE P802.22/ D0.3, May 2007.

[3] A. Ghasemi and E.S. Sousa, "Spectrum Sensing in Cognitive Radio Networks: Requirements, Challenges and Design Tradeoffs," IEEE Communications Magazine, April 2008, pp. 32-39.

[4] H. Arslan, Cognitive Radio, Software Defined Radio, and Adaptive Wireless Systems, Springer, 2007.

[5] Y. Zeng, C-L Koh, and Y-C Liang, "Maximum Eigenvalue Detection: Theory and Application," IEEE ICC2008, pp.4160-4164, 2008.

[6] A. Ghasemi and E. S. Sousa, "Spectrum Sensing in Cognitive Radio Networks: The Cooperation-Processing Trade-Off," Wiley Wireless Commun. and Mobile Comp. Special Issue on Cognitive Radio, Software-Defined Radio, and Adaptive Wireless Systems, vol. 7, no. 9, Nov. 2007, pp. 1049-60.

[7] J. Ma, G. Zhao, Ye Li, "Soft Combination and Detection for Spectrum Sensing in Cognitive Radio Networks," IEEE Trans. On Wireless Commun., vol.7, no.11, November 2008, pp.4502-4507.

[8] S. Shellhammer et al., Spectrum Sensing Simulation Model, IEEE 802.22-06/0028r4, March 2006.

[9] T. Rappaport, Wireless Communications: Principles and Practice, Prentice Hall, 1996.
[10] A. Ghasemi and E.S. Sousa, "Optimization of spectrum sensing for opportunistic spectrum access in cognitive radio networks," IEEE CCNC, Jan. 2007, pp.1022-1026.

[11] Y.C. Liang, Y. Zeng, E. Peh, et al., "Sensing-throughput tradeoff for cognitive radio networks," IEEE Trans. On Wireless Commun. vol. 7, no. 4, April 2008, pp.1326 1337.

[12] B-J Kang et al., "Sensing Optimization for a Receiver Structure in Cognitive Radio Systems," KIMICS, vol. 9, no. 1, February 2011, pp.27-31.

[13] FCC, Second Report and Order and Memorandum Opinion and Order in the Matter of Unlicensed Operation in the TV Broadcast Bands, Additional Spectrum for Unlicensed Device Below $900 \mathrm{MHz}$ and in the $3 \mathrm{GHz}$ Band, Document 08-260, November 2008.

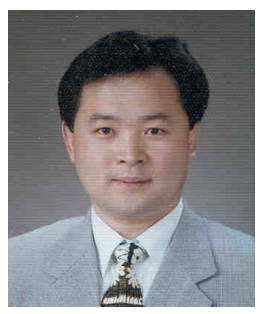

Bub-Joo Kang received the B.S. degree in electronic engineering from Kyunghee University, Seoul, Korea in 1983, and the M.S. and Ph.D. degrees in electronic engineering from Yonsei University, Seoul, Korea in 1985 and 1996, respectively. From 1988 to 2001, he worked for ETRI and served as a principal member of engineering staff at the Modem Technology Department, managing the project of design and development of radio modem technology for IMT2000. He is currently an associate professor in department of information and communications engineering, Dongguk University, Kyungbuk, Korea. His current research interests include cognitive radio, spectrum sensing, OFDM, CDMA, etc. 